\title{
Dinâmica espaço-temporal de flebotomíneos (Diptera, Psychodidae) do município de Santa Quitéria, área de cerrado do Estado do Maranhão, Brasil
}

\author{
Alexsandra M. C. B. Martin ${ }^{1} \&$ José M. Macário Rebêlo
}

1. Departamento de Biologia, Universidade Federal do Maranhão, Avenida dos Portugueses s/n, Campus do Bacanga, São Luís.

2. Laboratório de Entomologia e Vetores, Departamento de Patologia, Universidade Federal do Maranhão, Praça Madre Deus 2, 65025560 São Luís, MA, Brasil. (macariorebelo@uol.com.br)

\begin{abstract}
Spatial-temporal dynamics of phlebotomine sandflies (Diptera, Psychodidae) in the municipality of Santa Quitéria, "cerrado" area, State of Maranhão, Brazil. The seasonal fluctuation, hourly frequency and occurrence of phlebotomine sandflies inside and around dwellings and in the cerrado area of the municipality of Santa Quitéria, State of Maranhão were studied. The specimens were collected between 6:00 pm and 6:00 am from May 1999 to April 2000 through CDC-type light traps. A total of 4,880 specimens belonging to 11 species were collected. The highest species richness and abundance were observed around dwellings (11 species, $50.1 \%$ of the specimens), followed by species collected inside dwellings ( 9 species, $34 \%)$ and in the cerrado ( 7 species, $15.8 \%)$. Two species were found during the rainy season, one during the dry season and eight during both seasons. High frequencies of Lutzomyia longipalpis Lutz \& Neiva, 1912 (88.4\%), L. evandroi Costa Lima \& Antunes, 1936 (83.6\%), L. lenti Mangabeira, 1938 (86\%), and L. shannoni Dyar, $1929(100 \%)$ were recorded during the dry season, whereas the frequencies of $L$. quinquefer Dyar, $1929(100 \%)$ and $L$. whitmani Antunes \& Coutinho, 1939 (75\%) were higher during the rainy period. Phlebotomine sandflies were collected throughout the night, but the times of higher frequency varied according to species, with peaks being observed for L. quinquefer (6:00 - 7:00 pm), L. goiana Martins, Falcão \& Silva, 1962 (6:00 - 9:00 pm), L. whitmani (7:00 - 9:00 pm), and L. longipalpis (8:00 - 9:00 pm) during sunset and in the first night hours, and for L. evandroi (9:00 - 11:00 pm and 3:00 - 5:00 am), L. longipalpis (1:00 - 3:00 am), and L. lenti (10:00 - 11:00 pm and 4:00 - 5:00 am) throughout the night and during dawn. The occurrence of phlebotomine sandflies in different environments, at different times and during different seasons is accompanied by the notification of severed cases of cutaneous and visceral leishmaniasis.
\end{abstract}

KEYWORDS. Lutzomyia, seasonal occurrence, hourly frequency, leishmaniasis.

RESUMO. A flutuação sazonal, a freqüência horária e a ocorrência dos flebotomíneos nos ambientes intradomiciliar, peridomiciliar e extradomiciliar (cerrado), no município de Santa Quitéria, Estado do Maranhão foram estudados. Os espécimes foram capturados entre 18 e 6 horas, de maio de 1999 a abril de 2000, com armadilhas luminosas tipo CDC. Foram coletados 4.880 espécimes de 11 espécies. A riqueza e abundância foram maiores no peridomicílio (11 espécies; $50,1 \%$ dos espécimes), seguido pelo intradomicílio ( 9 espécies, $34 \%$ ) e cerrado (7 espécies, 15,8\%). Duas espécies foram encontradas na estação chuvosa, uma na estiagem e oito em ambas estações. Na estiagem foram registradas elevadas freqüências de Lutzomyia longipalpis Lutz \& Neiva, 1912 (88,4\%), L. evandroi Costa Lima \& Antunes, 1936 (83,6\%), L. lenti Mangabeira, 1938 (86\%) e L. shannoni Dyar, 1929 (100\%), enquanto as freqüências de L. quinquefer Dyar, $1929(100 \%)$ e L. whitmani Antunes \& Coutinho, 1939 (75\%) foram maiores no período chuvoso. Os flebotomíneos foram encontrados a noite inteira, mas os horários de maior freqüência variaram de acordo com a espécie, observando-se picos no crepúsculo vespertino e primeiras horas da noite: L. quinquefer $(18 \mathrm{~h}-19 \mathrm{~h})$; L. goiana Martins, Falcão \& Silva, $1962(18 \mathrm{~h}-21 \mathrm{~h}) ;$ L. whitmani $(19 \mathrm{~h}-21 \mathrm{~h})$ e L. longipalpis $(20 \mathrm{~h}-21 \mathrm{~h})$ e ao longo da noite e no crepúsculo matutino: L. evandroi $(21 \mathrm{~h}-23 \mathrm{~h} \mathrm{e} 3 \mathrm{~h}-5 \mathrm{~h}), L$ longipalpis $(1 \mathrm{~h}-3 \mathrm{~h})$, L. lenti $(22 \mathrm{~h}-23 \mathrm{~h}$ e $4 \mathrm{~h}-5 \mathrm{~h})$. A ocorrência dos flebotomíneos nos diferentes ambientes, horários e estações vem sendo acompanhada por notificações de vários casos de leishmanioses cutânea e visceral.

PALAVRAS-CHAVE. Lutzomyia, ocorrência sazonal, freqüência horária, leishmanioses.

O Estado do Maranhão situa-se entre três biomas de grande importância na distribuição dos flebotomíneos e na epidemiologia das leishmanioses. Por um lado, está a floresta amazônica e pelo outro, os cerrados do Brasil Central e as caatingas nordestinas (IBGE, 1984). Em função dessa posição e das variações fisiográficas e climáticas, o estado apresenta uma variedade de ecossistemas.

A distribuição dos flebotomíneos de certa forma reproduz esta situação. No lado oeste, que faz fronteira com o Estado do Pará, ocorrem, por exemplo, Lutzomyia umbratilis Ward \& Fraiha, 1977 e L. complexa (Mangabeira, 1941) (REBÊLo et al., 2000a, b), ausentes na parte nordestina, a leste, limítrofe com o Piauí, onde se encontram L. cortelezzii (Brèthes, 1923), L. lenti Mangabeira, 1938 e L. intermedia (Lutz \& Neiva, 1912), sendo muito comum a presença de L. longipalpis Lutz \& Neiva, 1912 ( ReBÊLo et al., 1999).

Em função desse padrão, a leishmaniose cutânea (LC) é mais evidente nas áreas amazônicas e a leishmaniose visceral (LV), no lado nordestino. Contudo, o intenso fluxo migratório e o incessante processo de modificação da cobertura vegetal vêm mudando os padrões epidemiológicos das leishmanioses (MARTINs et al., 2004), de modo que atualmente podem-se encontrar casos de ambas infecções em todo o estado. A continuidade desse processo pode selecionar espécies de flebotomíneos com capacidade de colonizar as áreas modificadas e até mesmo o ambiente peridomiciliar rural (CARVAlho et al., 2000; RebêLo et al., 1999; 2000a, b) e semi-urbana (ARAúJo et al., 2000). A transmissão dos primeiros casos de LC registrados no estado estava 
associada com o ambiente florestal (SiLva et al., 1979). Os estudos atuais mostram que há fortes indícios de que a transmissão da infecção humana esteja acontecendo também no ambiente domiciliar e peridomiciliar (PAssos et al., 2003).

Os estudos realizados no Maranhão indicam que várias espécies de flebotomíneos ocorrem o ano inteiro, outras se sucedem ao longo do ano, mas a freqüência de indivíduos, com raras exceções (ReBêLo et al., 2000b), aumenta na estação chuvosa em matas secundárias (BARRos et al., 2000) e primárias (REBÊLo et al., 2000b), assim como em povoados rurais (CARVALHO et al., 2000) e semi-urbanos (ARAÚJo et al., 2000). Esses mesmos estudos demonstraram que os flebotomíneos ocorrem a noite inteira ou em determinados horários a depender da espécie e do ambiente; contudo, picos no crepúsculo vespertino e nas primeiras horas da noite são os mais comuns.

Neste trabalho estudaram-se a variação sazonal, a freqüência horária e a ocorrência dos flebotomíneos nos ambientes domiciliar, peridoméstico e silvestre, com o intuito de ampliar não só os conhecimentos acerca da ecologia desses vetores, como também o entendimento da epidemiologia das leishmanioses numa área de cerrado com assentamento humano.

\section{MATERIAL E MÉTODOS}

O estudo foi realizado no município de Santa Quitéria, Maranhão, localizado na zona fisiográfica do Baixo Parnaíba, com uma área de $2.475 \mathrm{~km}^{2}$. Limita-se ao norte com Barreirinhas e São Bernardo, ao sul com Anapurus e Brejo, a leste com o Estado do Piauí e a oeste com Urbano Santos.

A cobertura vegetal resulta da mistura de espécies de mata perenifólia, da caatinga e do cerrado (IBGE, 1984), a qual se deve à grande complexidade climática e a possíveis diferenças pedológicas, com predomínio de solos areno-quartzosos e presença de solos concrecionários lateríticos e solos hidromórficos. As espécies da mata apresentam acentuada deciduidade, sendo comuns o pau d'arco (Tabebuia seratifolia Vahlnich.) (Bignoniaceae) e a murta (Mouriri guianensis Aubl) (Melastomataceae). Entre as espécies do cerrado, distingue-se o pau-terra (Qualea grandiflora Mart.) (Vochysiaceae) e o bacuri (Platonia insignis Mart.) (Clusiaceae). Da caatinga, são mais comuns o sabiá (Mimosa caesalpinaefolia Benth) e a jurema (Mimosa hostilis Benth) (Fabaceae). O clima predominante da área é o quente semi-árido, com índices pluviométricos anuais de $1.600 \mathrm{~mm}$. Os meses mais chuvosos são geralmente os de janeiro a maio, responsáveis por cerca de $73,3 \%$ do total anual. A temperatura varia muito pouco ao longo do ano (cerca de $3{ }^{\circ} \mathrm{C}$ ), sendo que a média anual está em torno de $27^{\circ} \mathrm{C}$

A área de estudo propriamente dita foi uma reserva particular de cerrado $\left(3^{\circ} 20^{\prime} 41^{\prime \prime} \mathrm{S}\right.$ e $\left.42^{\circ} 52^{\prime} 07^{\prime \prime} \mathrm{W}\right)$ pertencente à Comercial e Agrícola Paineiras Ltda, com uma área total de aproximadamente 45.000 ha, situada no povoado de Baixão. Essa área foi selecionada, sobretudo, por apresentar glebas de cerrado stricto sensu, representando o ambiente silvestre (natural) de ocorrência dos flebotomíneos e por estar incluída no pólo de produção das leishmanioses (LC e LV).

Os locais selecionados para as coletas dos insetos foram o interior do cerrado e, a $500 \mathrm{~m}$ distante deste, uma habitação, construída de adobo e coberta de palha, à semelhança da maioria das habitações do povoado de Baixão. O galinheiro ( $5 \mathrm{~m}$ da casa) representou o peridomicílio, e o dormitório, o intradomicílio. Na ocasião do estudo não foi possível a realização de réplica nos respectivos ambientes, em função do difícil acesso, distâncias entre as residências e os custos da pesquisa.

Os flebotomíneos foram capturados de maio de 1999 a abril de 2000, uma vez por mês, das $18 \mathrm{~h}$ às $6 \mathrm{~h}$, com três armadilhas luminosas tipo CDC (Center for Disease Control and Prevention), distribuídas, cada uma, no seu respectivo ambiente: cerrado, peridomicílio e intradomicílio, a $1,5 \mathrm{~m}$ de altura, sempre nos mesmos locais, seguindo o método utilizado por ReBêLo et al. (2000a, b). Cada armadilha funcionou 12 horas consecutivas, resultando em 432 horas trabalhadas. Os exemplares capturados eram postos em câmaras de acetato de etila e transportados para o laboratório, onde eram montados em Berlese e identificados.

Para análise das diferenças nas proporções de indivíduos capturados entre os ambientes, utilizou-se o teste Qui-Quadrado. Para a análise da abundância relativa (\%) das espécies predominantes e seus limites de confiança, foi utilizado o método de Kato et al. apud LAROCA (1995). As espécies foram consideradas dominantes quando seu limite de confiança inferior foi maior que o limite superior para espécies ausentes. A diversidade nos ambientes foi calculada pelo índice de Shannon-Wiener. Para medida de similaridade foi utilizado o índice de Morisita-Horn, para dados quantitativos. Foi aplicada a correlação não-paramétrica de Spearman entre o número de espécimes encontrados e as médias da precipitação pluviométrica, medidos ao longo do ano. Calculou-se também o índice de Constância dos flebotomíneos, pela fórmula: $\mathrm{C}=\mathrm{P}$ X $100 / \mathrm{N}$; onde $\mathrm{P}=$ número de coletas com a espécie estudada e $\mathrm{N}=$ número total de coletas efetuadas (Silveira Netto et al., 1976).

\section{RESULTADOS}

Foram encontradas 11 espécies de Lutzomyia França, 1924: L. longipalpis Lutz \& Neiva, 1912; L. evandroi Costa Lima \& Antunes, 1936; L. lenti Mangabeira, 1938; L. goiana Martins, Falcão \& Silva, 1962; L. whitmani Antunes \& Coutinho, 1939; L. shannoni Dyar, 1929; L. quinquefer Dyar, 1929; L. termitophila Martins, Falcão \& Silva, 1971; L. punctigeniculata Floch \& Abonnenc, 1944; L. sordellii Shannon \& Del Pont, 1927 e uma espécie não determinada, da qual só foram encontradas fêmeas, podendo ser tanto L. wellcomei Fraiha, Shaw \& Lainson, 1971 como L. complexa (Mangabeira, 1941).

No total, foram capturados 4.880 espécimes, com o predomínio de L. longipalpis $(78,07 \%)$, L. evandroi $(10,7 \%)$, L. lenti $(4,81 \%)$, L. goiana $(4,07 \%)$ e L. whitmani $(1,06 \%)$ (Tab. I). Quando a análise foi feita por ambiente, obteve-se a seguinte ordem de dominância: cerrado - $L$. longipalpis $(67,6 \%)$, L. evandroi $(24,6 \%)$, L. goiana 
$(6,1 \%)$ e L. lenti $(0,9 \%)$; peridomicílio - L. longipalpis $(92,1 \%)$, L. goiana $(2,6 \%)$, L. evandroi $(1,7 \%)$, L. lenti $(1,3 \%), L$. whitmani $(1,0 \%)$ e L. quinquefer $(0,6 \%)$; e intradomicílio - L. longipalpis $(62,4 \%)$, L. evandroi $(18 \%)$, L. lenti $(11,9 \%)$, L. goiana $(5,2 \%)$, L. whitmani $(1,5 \%) \mathrm{e}$ L. shannoni $(0,8 \%)$.

De um modo geral, os machos predominaram $(54,8 \%)$ sobre as fêmeas $(45,2 \%)$. Tal resultado foi influenciado pelo padrão de abundância dos machos de três espécies: L. longipalpis, que contribuiu com 54\% dos espécimes, representando mais de dois mil exemplares; e de L. lenti e L. shannoni, que contribuíram apenas com machos. Nas demais espécies as fêmeas predominaram, com destaque para L. evandroi $(54,5 \%)$, L. goiana (56\%) e L. quinquefer $(86,7 \%)$.

Todas as espécies estiveram presentes no peridomicílio, sendo que nove adentraram nas habitações e apenas sete foram encontradas no ambiente silvestre (Tab. I). A diversidade foi maior no intradomicílio $\left(\mathrm{H}^{\prime}=\right.$ $1,12)$ que na mata $\left(H^{\prime}=0,86\right)$ e peridomicílio $\left(H^{\prime}=0,41\right)$. A porcentagem de similaridade foi maior entre o peridomicílio e a mata $(\% \mathrm{~S}=73,33 \%)$ que entre o intra- e o peridomicílio $(\% \mathrm{~S}=69,57 \%)$ e entre o intradomicílio e a mata $(\% \mathrm{~S}=36,82 \%)$. A frequiência de indivíduos foi maior no peridomicílio (50,14\%) que no intradomicílio $(34,01 \%)$ e na mata $(15,81 \%)$.

Os espécimes estiveram presentes em todos os meses do ano, mas a freqüência foi maior em outubro $(36,6 \%)$, setembro $(13,2 \%)$ e dezembro $(22,1 \%)$, na estação de estiagem, e abril (10,6\%), na estação chuvosa. Durante o ano de estudo, a precipitação pluviométrica atingiu $1.600 \mathrm{~mm}$ concentrados na estação chuvosa (88\%), mas

Tabela I. Espécies de flebotomíneos encontradas no ambiente silvestre (cerrado), intra- e peridomiciliar, no município de Santa Quitéria, MA, Brasil, de maio de 1999 a abril de 2000 (M, machos; F, fêmeas).

\begin{tabular}{lrrrrrrrrrrrrr}
\hline Ambientes & \multicolumn{4}{c}{ Cerrado } & \multicolumn{3}{c}{ Peridomicílio } & \multicolumn{3}{c}{ Intradomicílio } & \multicolumn{1}{c}{ Total } \\
\hline Espécies/Sexos & M & F & Total & M & F & Total & M & F & Total & M & \multicolumn{1}{c}{ F } & $\mathrm{n}^{0}$ & $\%$ \\
\hline L. evandroi & 101 & 89 & 190 & 18 & 24 & 42 & 122 & 176 & 298 & 241 & 289 & 530 & 10,86 \\
L. goiana & 19 & 28 & 47 & 41 & 23 & 64 & 27 & 60 & 87 & 87 & 111 & 198 & 4,07 \\
L. lenti & 7 & - & 7 & 31 & - & 31 & 197 & - & 197 & 235 & - & 235 & 4,81 \\
L. longipalpis & 218 & 304 & 522 & 1.451 & 803 & 2.254 & 388 & 648 & 1.036 & 2.057 & 1.755 & 3.812 & 78,07 \\
L. punctigeniculata & - & 2 & 2 & - & 1 & 1 & - & - & - & - & 3 & 3 & 0,10 \\
L. quinquefer & - & - & - & 2 & 12 & 14 & - & 1 & 1 & 2 & 13 & 15 & 0,30 \\
L. shannoni & - & - & - & 9 & - & 9 & 14 & - & 14 & 23 & - & 23 & 0,47 \\
L. sordellii & - & - & - & - & 1 & 1 & - & - & - & - & 1 & 1 & 0,02 \\
L. termitophila & - & 2 & 2 & 1 & 3 & 4 & - & 1 & 1 & 1 & 6 & 7 & 0,14 \\
L. complexa/wellcomei & - & - & - & - & 3 & 3 & - & 1 & 1 & - & 4 & 4 & 0,10 \\
L. whitmani & - & 2 & 2 & 14 & 11 & 25 & 14 & 11 & 25 & 28 & 24 & 52 & 1,06 \\
\hline $\mathrm{N}^{\mathrm{o}}$ de indivíduos & 345 & 427 & 772 & 1.567 & 881 & 2.448 & 762 & 898 & 1.660 & 2.674 & 2.206 & 4.880 & 100,00 \\
\hline \% de indivíduos & 44,7 & 55,3 & 15,8 & 64,0 & 36,0 & 50,2 & 45,9 & 54,1 & 34,0 & 54,8 & 45,2 & 100,0
\end{tabular}

Tabela II. Freqüência mensal das espécies de flebotomíneos encontradas nos ambientes silvestre (cerrado), intra- e peridomiciliar, no município de Santa Quitéria, MA, Brasil, de maio de 1999 a abril de 2000.

\begin{tabular}{|c|c|c|c|c|c|c|c|c|c|c|c|c|}
\hline \multirow{2}{*}{$\begin{array}{l}\text { Estações } \\
\text { Espécies/Meses }\end{array}$} & \multicolumn{6}{|c|}{ Chuvosa } & \multicolumn{6}{|c|}{ Estiagem } \\
\hline & $\mathrm{J}$ & $\mathrm{F}$ & $\mathrm{M}$ & $\mathrm{A}$ & $\mathrm{M}$ & $\mathrm{J}$ & $\mathrm{J}$ & $\mathrm{A}$ & $\mathrm{S}$ & $\mathrm{O}$ & $\mathrm{N}$ & $\mathrm{D}$ \\
\hline L. evandroi & 20 & 5 & 1 & 56 & 3 & 2 & 8 & 7 & 212 & 44 & 41 & 131 \\
\hline L. goiana & 32 & 6 & 5 & 34 & 13 & 44 & 10 & 8 & 23 & 5 & 6 & 12 \\
\hline L. longipalpis & 20 & 7 & 8 & 392 & 5 & 10 & 10 & 37 & 241 & 1.725 & 454 & 903 \\
\hline L. lenti & 6 & 7 & - & 19 & - & 1 & 2 & 1 & 146 & 9 & 11 & 33 \\
\hline L. punctigeniculata & - & - & - & 1 & 1 & - & 1 & - & - & - & - & - \\
\hline L. quinquefer & 1 & - & 1 & - & - & 13 & - & - & - & - & - & - \\
\hline L. shannoni & - & - & - & - & - & - & - & - & 23 & - & - & - \\
\hline L. sordellii & - & - & - & 1 & - & - & - & - & - & - & - & - \\
\hline L. termitophila & 3 & - & - & 1 & - & - & 1 & - & 1 & - & - & 1 \\
\hline L. whitmani & 1 & 3 & 14 & 12 & 1 & 8 & 1 & 10 & - & 2 & - & - \\
\hline L. complexa/wellcomei & - & 3 & - & - & - & - & - & 1 & - & - & - & - \\
\hline $\mathrm{N}^{0}$ de espécies & 7 & 6 & 5 & 8 & 5 & 6 & 7 & 6 & 6 & 5 & 4 & 5 \\
\hline $\mathrm{N}^{\circ}$ de indivíduos & 83 & 31 & 29 & 516 & 23 & 78 & 33 & 64 & 646 & 1.785 & 512 & 1.080 \\
\hline $\mathrm{N}^{\circ}$ percentuais & \multicolumn{6}{|c|}{15,6} & \multicolumn{6}{|c|}{84,4} \\
\hline $\begin{array}{l}\text { Precipitação } \\
\text { pluviométrica }(\mathrm{mm})\end{array}$ & 210 & 250 & 350 & 260 & 220 & 50 & 55 & 25 & 10 & 30 & 40 & 100 \\
\hline
\end{tabular}


o número de indivíduos foi maior na estação de estiagem $(84,4 \%)$ (Tab. II). Em função desse padrão, não houve correlação entre os valores da precipitação pluviométrica e a freqüência mensal de $L$. evandroi $(\mathrm{r}=-0,4056 ; \mathrm{t}=$ $1,4032 ; \mathrm{p}=0,1908)$, L. goiana $(\mathrm{r}=0,0105 ; \mathrm{t}=0,0333 ; \mathrm{p}=$ $0,9741)$, L. longipalpis $(\mathrm{r}=-0,4939 ; \mathrm{t}=-1,7961 ; \mathrm{p}=0,1026)$, L. lenti $(\mathrm{r}=-0,3038 ; \mathrm{t}=-1,0266 ; \mathrm{p}=0,3288)$ e L. whitmani $(\mathrm{r}=0,4469 ; \mathrm{t}=1,5795 ; \mathrm{p}=0,1452)$. A freqüência mensal das espécies variou de quatro (novembro) a oito (abril), ao longo do ano.

Neste estudo, L. longipalpis, L. evandroi e $L$. goiana foram as únicas a exibirem comportamento de espécies anuais, sendo juntamente com L. lenti e $L$. whitmani classificadas como constantes, por estarem presentes na área em mais de $50 \%$ das coletas. Seguindo o mesmo critério de análise, L. punctigeniculata, L. quinquefer e L. termitophila foram consideradas acessórias, visto que ocorreram entre $25 \%$ e $50 \%$ das coletas, enquanto L. shannoni, L. sordellii e L. complexal wellcomei comportaram-se como espécies acidentais por terem aparecido em menos de $25 \%$ das coletas.

De um modo geral, os flebotomíneos distribuíramse em todos os horários (Tab. III), mas a freqüência foi significativamente maior $\left(\chi^{2}=16,752 ; \mathrm{GL}=1 ; \mathrm{p}=0,0000\right)$ na primeira metade da noite $(54,1 \%)$. Tal padrão foi observado também para as seis espécies mais freqüentes: L. evandroi $(54,7 \%)$, L. goiana $(71,2 \%)$, L. longipalpis (52\%), L. lenti $(62,1 \%)$, L. whitmani $(78,8 \%)$ e L. shannoni $(73,9 \%)$. Quanto aos picos de freqüência, estes foram observados no crepúsculo vespertino e primeiras horas da noite: L. quinquefer (18 h - 19h); L. goiana $(18 \mathrm{~h}-21 \mathrm{~h})$ e L. whitmani (19 h - 21h); e ao longo da noite e no crepúsculo matutino: L. evandroi $(21 \mathrm{~h}-23 \mathrm{~h}$ e $3 \mathrm{~h}-5 \mathrm{~h})$, L. longipalpis $(20 \mathrm{~h}-21 \mathrm{~h}$ e $1 \mathrm{~h}-3 \mathrm{~h})$, L. lenti $(22 \mathrm{~h}-23 \mathrm{~h}$ e $4 \mathrm{~h}-5 \mathrm{~h})$.

\section{DISCUSSÃO}

Os dados obtidos neste estudo ampliaram o conhecimento da fauna de flebotomíneos do nordeste maranhense, elevando o registro atual para 15 espécies.
Foram encontradas cinco espécies até então desconhecidas no nordeste do Estado, do qual faz parte o município de Santa Quitéria: L. quinquefer, L. sordellii, L. punctigeniculata, L. shannoni e L. wellcomei/L. complexa. Por outro lado, quatro espécies já conhecidas naquela região não foram encontradas neste estudo: $L$. cortelezii, L. intermedia, L. longipennis e L. squamiventris (REBÊLo et al., 1999). Em relação à identificação de L. wellcomei/L. complexa, as fêmeas dessas duas espécies são morfologicamente idênticas, sendo impossível separá-las sem a presença de machos, a não ser por meio de técnicas mais refinadas, como de biologia molecular (READY et al., 1991). Lutzomyia wellcomei ocorre na Amazônia paraense, sendo encontrada na Serra dos Carajás (WARD et al., 1973), onde transmite Leishmania braziliensis (RYAN et al., 1987). O Nordeste tem registro de ocorrência na mata serrana do Ceará (READy et al., 1984) e na zona da mata de Pernambuco (BRANDÃo-Filho et al., 1998). No Maranhão foi detectada na ilha de São Luís, nos meses de janeiro e fevereiro, associada com capoeira e domicílio rural. A distribuição de L. complexa está limitada à Bacia Amazônica, incluindo os estados de Rondônia (Bianchardi et al., 1982), Mato Grosso (Lainson et al., 1976) e Pará (FrAIHA et al., 1978). Mais recentemente foram coletados machos e fêmeas, nas estações de estiagem e chuvosa, no município de Paragominas, PA (REBÊLo \& Oliveira-Pereira, 2001). No que concerne ao Maranhão, esta espécie foi detectada nas matas amazônicas de Buriticupu, não tendo registro para o nordeste do Estado. Pelo padrão de distribuição de ambas espécies, a probabilidade das fêmeas encontradas neste estudo pertencerem a L. wellcomei seria maior.

A riqueza de espécies de flebotomíneos registrada no município de Santa Quitéria foi equivalente à dos municípios de Paço do Lumiar (10 espécies), Raposa (11), São José de Ribamar (13) e Codó (10), situados na zona semi-úmida de transição entre o clima quente e úmido e o semi-árido, de vegetação mais seca, característico do leste do Maranhão (ARAúJo et al., 2000; BARRos et al., 2000; CARvalho et al., 2000; ReBÊLo et al., 1999), porém foi

Tabela III. Freqüência horária das espécies de flebotomíneos encontrados nos ambientes silvestre (cerrado) e intra- e peridomiciliar, no município de Santa Quitéria, MA, Brasil, de maio de 1999 a abril de 2000.

\begin{tabular}{|c|c|c|c|c|c|c|c|c|c|c|c|c|}
\hline Espécies/Horários & 18 & 19 & 20 & 21 & 22 & 23 & 00 & 01 & 02 & 03 & 04 & 05 \\
\hline & 19 & 20 & 21 & 22 & 23 & 24 & 01 & 02 & 03 & 04 & 05 & 06 \\
\hline L. evandroi & 25 & 54 & 46 & 63 & 63 & 39 & 21 & 38 & 43 & 64 & 62 & 12 \\
\hline L. goiana & 36 & 25 & 27 & 21 & 19 & 13 & 7 & 21 & 8 & 4 & 10 & 7 \\
\hline L. longipalpis & 229 & 361 & 460 & 394 & 245 & 297 & 278 & 538 & 412 & 315 & 237 & 46 \\
\hline L. lenti & 15 & 30 & 25 & 11 & 41 & 24 & 9 & 13 & 15 & 13 & 32 & 7 \\
\hline L. punctigeniculata & & & & 1 & & & 1 & & & 1 & & \\
\hline L. quinquefer & 10 & 4 & 1 & & & & & & & & & \\
\hline L. shannoni & 1 & 3 & 6 & & 7 & & 1 & 3 & 2 & & & \\
\hline L. sordellii & & & & & 1 & & & & & & & \\
\hline L. termitophila & 1 & & 1 & 1 & 1 & & 1 & & & 2 & & \\
\hline L. whitmani & 3 & 11 & 9 & 7 & 5 & 6 & & 3 & 1 & 2 & 4 & 1 \\
\hline L. complexa/wellcomei & & & & & & & & 2 & 1 & & 1 & \\
\hline $\mathrm{N}^{0}$ de espécies & 8 & 7 & 8 & 7 & 8 & 5 & 7 & 7 & 7 & 7 & 6 & 5 \\
\hline $\mathrm{N}^{\circ}$ de indivíduos & 320 & 488 & 575 & 498 & 382 & 379 & 318 & 618 & 482 & 401 & 346 & 73 \\
\hline
\end{tabular}


inferior àquela observada em Buriticupu (38 espécies), no lado amazônico (REBÊLo et al., 2000a).

A maioria das espécies foi representada por poucos indivíduos, talvez pela ausência de suas fontes alimentares sangüíneas, de criadouros e/ou de abrigos nas proximidades das armadilhas. Considerando que os flebotomíneos eram capturados em locais fixos, as espécies mais estritas a determinados ecótopos podem ter sido representadas apenas por alguns indivíduos que utilizavam aqueles pontos ocasionalmente. Soma-se a estes aspectos a eficácia das armadilhas $\mathrm{CDC}$, cujo raio de atração não excede $5 \mathrm{~m}$ (KILLICK-KENDRICK et al., 1985). De qualquer modo, essas espécies aparentemente raras na área podem vir a ter sua densidade aumentada em funções de alterações nas comunidades.

Todas as espécies encontradas neste estudo estavam presentes no peridomicílio; entre as mais freqüentes, destacam-se L. longipalpis e L. whitmani, por serem eficientes vetores da LV e da LC, respectivamente, e por terem adentrado no domicílio. Este aspecto epidemiológico é importante, pois pode sugerir a possibilidade de transmissão dessas doenças também no ambiente domiciliar.

A dominância de L. longipalpis mostra que ela se encontra bastante adaptada em todos os ambientes, o que explica a ocorrência de 137 casos de calazar, na última década, na região estudada. Todavia, a peridomiciliação do vetor nessas áreas rurais pouco desenvolvidas vem sendo acompanhada pelas alterações das matas, produto da ação antrópica (MARTINS et al., 2004). Estas alterações, juntamente com a presença constante e em elevada concentração de animais domésticos, acabam favorecendo a manutenção do vetor nas proximidades dos domicílios (PAssos et al., 2003), afetando os padrões epidemiológicos das leishmanioses (Dourado et al., 1989). No Maranhão, já é bem conhecida a proliferação de L. longipalpis em focos peridomésticos e periurbanos de LV, mas se essa tendência se intensificar com $L$. whitmani e outras espécies, a mudança no padrão epidemiológico da LC será iminente. A presença freqüente de L. whitmani no peridomicílio em várias partes do Maranhão já é bem conhecida (REBÊLo et al., 1999, 2000a, b), o que explica a marca de 5.361 casos de LC somente no nordeste do Estado, na última década. A transmissão desses casos deve-se certamente a esta espécie, sem desprezar a participação de outras potencialmente capazes de transmitir a infecção. São exemplos: $L$. lenti, a mais comum nessa região (leste) do estado (ReBÊLo et al., 1999), estando ausente na maioria dos estudos realizados no lado oeste, onde o clima é mais úmido; $L$. quinquefer, que segue o mesmo padrão; e L. evandroi e L. goiana, que apresentam ampla distribuição no Maranhão, estendendo-se até a Pré-Amazônia, onde são abundantes.

Nesse estudo, dois aspectos chamaram a atenção quanto à variação sazonal. Primeiro, à semelhança de outros estudos realizados no Maranhão (ARAÚJo et al., 2000; Barros et al., 2000; CARVAlHo et al., 2000; ReBÊLO et al., 2000b), poucas espécies foram encontradas rigorosamente em todos os meses; a maioria se sucedeu ao longo do ano; e somente $L$. evandroi, L. goiana, $L$. longipalpis comportaram-se como espécies anuais. Tal comportamento foi exibido também por $L$. whitmani e $L$. evandroi nos municípios de Buriticupu (ReBÊLo et al., 2001a, b) e em São José de Ribamar (CARVAlHo et al., 2000). Segundo, diferentemente dos outros estudos realizados no Maranhão, que encontraram maior abundância de indivíduos na estação chuvosa, neste estudo o predomínio se deu no período de estiagem devido, sobretudo, ao padrão de abundância de $L$. evandroi, L. longipalpis e L. lenti em setembro e das duas primeiras de outubro a dezembro. Comportamento similar só foi observado na localidade de Lagoas, município de Buriticupu, pela alta freqüência de $L$. whitmani, L. migonei e L. serrana em outubro (REBÊLo et al., 2001a). Essas observações mostram claramente que a flutuação sazonal na freqüência de indivíduos e de espécies (anuais, acessórias e acidentais) pode variar na mesma área, assim como geograficamente, e devem ser consideradas quando da elaboração de estratégia de controle das leishmanioses.

Quanto à atividade horária, as espécies apresentaram comportamento bastante eclético, sendo encontradas nos crepúsculos vespertino e matutino e durante a noite, com picos em horários distintos. Nas áreas tropicais elas tendem a ocorrer com maior freqüência nos primeiros horários da noite (GomEs et al., 1983; GALATI et al., 1996). Já no subtrópico (Paraná), picos de atividade podem ser observados próximo ao amanhecer (TEODORO et al., 1991). Nesse estudo, L. goiana, L. whitmani e $L$. quinquefer apresentaram maior freqüência no crepúsculo vespertino, ao contrário de L. longipalpis, L. evandroi $\mathrm{e}$ L. lenti, cuja distribuição foi mais regular ao longo da noite, corroborando as observações efetuadas na ilha de São Luís (REBÊLo, 2001).

Este estudo, além de ampliar o conhecimento da fauna flebotomínica do Maranhão, apresentou dados sobre espécies até então desconhecidas para o nordeste do Estado, confirmou o predomínio de L. longipalpis e registrou a presença de $L$. whitmani, que vem sendo encontrada em todas as localidades estudadas no Maranhão. Em relação a esta última, estimula-se a realização de estudos mais aprofundados, no sentido de se conhecer melhor as suas diversas populações e detectarse, por exemplo, a existência de variações morfológicas e/ ou a nível biomolecular. Finalmente, o padrão de distribuição dos flebotomíneos nos diferentes horários, estações e ambientes deve ser considerado para subsidiar os órgãos de saúde no controle vetorial e na prevenção das leishmanioses no município de Santa Quitéria.

Agradecimentos. À Comercial e Agrícola Paineiras Ltda., por permitir a realização deste trabalho em sua propriedade.

\section{REFERÊNCIAS BIBLIOGRÁFICAS}

Araújo, J. C.; Rebêlo, J. M. M.; Carvalho, M. L. \& Barros, V. L. L. 2000. Composição dos flebotomíneos (Diptera, Psychodidae) do município da Raposa-MA, Brasil. Área endêmica de leishmanioses. Entomología y Vectores 7(1):33-47.

Barros, V. L.; Rebêlo, J. M. M. \& Silva, F. S. 2000. Flebotomíneos (Diptera, Psychodidae) de capoeira do município do Paço do Lumiar, Estado do Maranhão, Brasil. Área endêmica de leishmanioses. Cadernos de Saúde Pública 16(1): 265-270.

Bianchardi, C. B.; Arias, J. R.; Freitas, R. A. \& Castelon, E. G. 
1982. The known geographical distribution of sand flies in the state of Rondônia, Brazil (Diptera, Psychodidae). Acta Amazonica 12(1):167-179.

Brandão-Filho, S. P.; Brito, M. E. F.; Martins, C. A. P.; Sommer, I. B.; Valença, H. F.; Almeida, F. A. \& Gomes, J. 1998. Leishmaniose tegumentar americana em centro de treinamento militar localizado na Zona da Mata de Pernambuco, Brasil. Revista da Sociedade Brasileira de Medicina Tropical 31(6):575-578.

Carvalho, M. L.; Rebêlo, J. M. M.; Araújo, J. C. \& Barros, V. L. L. 2000. Aspectos ecológicos dos flebotomíneos (Diptera, Psychodidae) do município de São José de Ribamar, MA, Brasil. Área endêmica de leishmanioses. Entomología y Vectores 7(1):19-32.

Dourado, M. I. C.; Noronha, C. V.; Alcantara, N.; Ichihara, M. Y. \& Loureiro, S. 1989. Epidemiologia da leishmaniose tegumentar americana e suas relações com a lavoura e o garimpo, em localidade do Estado da Bahia (Brasil). Revista de Saúde Pública 23(1):2-8.

Fraiha, H.; Ward, R. D.; Shaw, J. J. \& Lainson, R. 1978. Fauna antropofílica de flebótomos da rodovia Transamazônica, Brasil. Boletin de la Oficina Sanitária Panamericana 84(2): 134-139.

Galati, E. A. B.; Nunes, V. L. B.; Dorval, M. E. C.; Oshiro, E. T.; Cristaldo, G.; Espíndola, M. A.; Rocha, H. C. \& Garcia, W. B. 1996. Estudo dos flebotomíneos (Diptera: Psychodidae), em área de leishmaniose tegumentar, no Estado de Mato Grosso do Sul, Brasil. Revista de Saúde Pública 30(2):115-128.

Gomes, A. de C.; Rabello, E. X.; Santos, J. L. F.; Galati, E. A. B. 1983. Aspectos ecológicos da leishmaniose tegumentar americana. 3- Observações naturais sobre o ritmo diário de atividade de Psychodopygus intermedius em ambiente florestal e extraflorestal. Revista de Saúde Pública 17(1):23-30.

IBGE (Instituto Brasileiro de Geografia e Estatística). 1984. Atlas do Maranhão. Rio de Janeiro, Governo do Estado do Maranhão. 104p.

Killick-Kendrick, R.; Wilkes, T. J.; Alexander, J.; Bray, R. S.; Rioux, J. A. \& BAilly, M. 1985. The distance of attraction of CDC light traps to phlebotomine sandflies. Annales de Parasitologie et Humaine Comparée 60(6):763-767.

Lainson, R.; Ward, R. D.; Young, D. G.; Shaw, J. J. \& Frahia, H. 1976. Preliminary entomological and parasitological studies in Humboldt, Aripuana, Mato Grosso State, Brazil. Acta Amazonica 6(Suppl. 1):55-60.

LAROCA, S. 1995. Ecologia: princípios e métodos. Petrópolis, Vozes. 197p.

Martins, L. M.; Rebêlo, J. M. M.; Santos, M. C. F. V.; Costa, J. M. L.; Silva, A. R. \& Ferreira, L. A. 2004. Ecoepidemiologia da leishmaniose tegumentar no Município de Buriticupu, Amazônia do Maranhão, Brasil, 1996 a 1998. Cadernos de Saúde Pública 20(3):735-743.

Passos, F. O.; Lorosa, E. S. \& Rebêlo, J. M. M. 2003. Fonte alimentar sangüínea e a peridomiciliação de Lutzomyia longipalpis (Lutz \& Neiva, 1912) (Psychodidae, Phlebotominae). Cadernos de Saúde Pública 19(5):109-118.

Ready, P. D.; Lainson, R. \& Shaw, J. J. 1984. Habitat and seasonality of Psychodopygus wellcomei help incriminate it as a vector of Leishmania braziliensis in Amazonia and northeast Brazil. Transactions of the Royal Society of Tropical Medicine and Hygiene 78(4):543-544.

Ready, P. D.; Lainson, R.; Shaw, J. J. \& Souza, A A. 1991. DNA probes for distinguishing Psychodopygus wellcomei from Psychodopygus complexa (Diptera: Psychodidae). Memórias do Instituto Oswaldo Cruz 86(1):41-49.

RebêLo, J. M. M. 2001. Freqüência horária e sazonalidade de Lutzomyia longipalpis (Diptera: Psychodidae: Phlebotominae) na Ilha de São Luís, Maranhão, Brasil. Cadernos de Saúde Pública 17(1):221-227.

Rebêlo, J. M. M. \& Oliveira-Pereira, Y. N. 2001. Flebotomíneos (Diptera, Psychodidae) de matas de terra firme e de várzea, do município de Paragominas, Estado do Pará, Brasil. Acta Amazonica 31(1):145-154.

Rebêlo J. M. M.; Oliveira, S. T. \& Silva, F. S. 2001a. Flutuação sazonal dos flebotomíneos (Diptera, Psychodidae) em área de colonização recente do município de Buriticupu, Amazônia maranhense, Brasil. Revista Brasileira de Entomologia 45(1): 11-16

Rebêlo, J. M. M.; Oliveira, S. T.; Barros, V. L. L. \& Silva, F. S. 2000a. Flebotomíneos (Diptera, Psychodidae) de Lagoas, município de Buriticupu, Amazônia maranhense. I - Riqueza e abundância relativa das espécies em área de colonização recente. Revista da Sociedade Brasileira de Medicina Tropical 33(1):11-19.

2000b. Flebotomíneos da Amazônia maranhense. IV Riqueza e abundância relativa das espécies em área de colonização antiga. Entomología y Vectores 7(1):61-72.

Rebêlo, J. M. M.; Oliveira, S. T.; Silva, F. S. \& Costa, J. M. L. 2001b. Sandflies (Diptera, Psychodidae) of the amazonia of Maranhão. V. Seasonal occurrence in ancient colonization area and endemic for cutaneous leishmaniasis. Revista Brasileira de Biologia 61(1):107-115.

Rebêlo, J. M. M.; Leonardo, F. S.; Costa, J. M. L.; Pereira, Y. N. O. \& Silva, F. S. 1999. Flebotomíneos (Diptera, Psychodidae) de área endêmica de leishmanioses na região dos cerrados, estado do Maranhão, Brasil. Cadernos de Saúde Pública 15(3):247-253.

Ryan, L.; Lainson, R. \& SHaw, J. J. 1987. Leishmaniasis in Brazil. XXIV. Natural flagellate infections of sandflies (Diptera: Psychodidae) in Pará State, with particular reference to the role of Psychodopygus wellcomei as the vector of Leishmania braziliensis in the Serra do Carajas. Transactions of the Royal Society of Tropical Medicine and Hygiene 81(3):353-359.

Silva, A. R.; Martins, G.; Melo, J. E. M.; Araújo, J. P. \& Mendes, M. L. 1979. Surto epidêmico de leishmaniose tegumentar americana ocorrido na colonização agrícola de Buriticupu (Estado do Maranhão), Brasil. Revista do Instituto de Medicina Tropical 21:43-50.

Silveira Netto, S.; Nakano, O.; Barbin, D. \& Villa Nova, N. A. 1976. Manual de ecologia dos insetos. São Paulo, Agronômica Ceres. 419p.

Teodoro, U.; La Salvia Filho, V. \& Lima, E. M.; Misuta, N. M.; Verginase, T.G. \& Ferreira, M. E. 1991. American cutaneous leishmaniasis: phlebotominae of the area of transmission in the North of Parana, Brazil. Revista de Saúde Pública 25(2):129-133.

Ward, R. D.; Shaw, J. J.; Lainson, R. \& Fraiha, H. 1973. Leishmaniasis in Brazil: VIII. Observations on the phlebotomine fauna of an area highly endemic for cutaneous leishmaniasis, in the Serra dos Carajás, Pará State. Transactions of the Royal Society of Tropical Medicine and Hygiene 67(2):174-183. 